\title{
Isolation and osteogenic differentiation of skeletal muscle-derived stem cells for bone tissue engineering
}

\author{
JUN ZOU, CHENXI YUAN, CHUNSHEN WU, CHENG CAO, QING SHI and HUILIN YANG
}

Department of Orthopaedic Surgery, The First Affiliated Hospital of Soochow University, Suzhou, Jiangsu 215006, P.R. China

Received May 14,2013; Accepted October 22, 2013

DOI: $10.3892 / \mathrm{mmr} .2013 .1758$

\begin{abstract}
The purpose of this study was to investigate the isolation and culture of muscle-derived stem cells (MDSCs) and their capability to differentiate into osteoblasts in vitro. Skeletal muscle tissue was obtained from double hind limbs of New Zealand white rabbits under sterile conditions and isolated by collagenase digestion. Following passages in basic medium, the primary cells were desmin (+), myosin (+) and CD105 (+). Differentiation of MDSCs was induced by osteogenic medium. Using a 3-(4,5-dimethylthiazol-2-yl)-2,5-diphenyl tetrazolium bromide assay, the differentiated cell population was found to proliferate faster than the undifferentiated. Alkaline phosphatase staining and alizarin red staining revealed that the differentiated cells were mineralized in vitro. Quantitative polymerase chain reaction assays also showed increased mRNA expression of osteogenic genes in differentiated cells. In conclusion, stem cells were successfully isolated and cultured from rabbit skeletal muscle tissue and were able to differentiate into osteoblasts following induction. These observations may indicate an ideal stem cell source for tissue engineering.
\end{abstract}

\section{Introduction}

In previous years, with the rapid development of molecular biology and tissue engineering, novel strategies on bone repair from basic research to clinical applications have been highly emphasized, in particular the role of the somatic stem cell. At present, studies of stem cells focus largely on bone marrow mesenchymal stem cells (BMSCs). In vitro and in vivo studies have confirmed that BMSCs have the ability to differentiate into osteoblasts, chondrocytes, adipocytes and neurons, as well as numerous other cell types $(1,2)$, which indicates a wide application prospect in tissue engineering. However, tradi-

Correspondence to: Dr Jun Zou, Department of Orthopaedic Surgery, The First Affiliated Hospital of Soochow University, 188 Shizi Street, Suzhou, Jiangsu 215006, P.R. China

E-mail: jzou@suda.edu.cn

Key words: stem cells, skeletal muscle, osteogenic differentiation, tissue engineering tional bone marrow acquisition requires anesthesia and often causes pain on puncture point, as well as harm to the physical and psychological health of patients. The in vitro purification rate of BMSCs is low at $\sim 1 \times 10$ cells $/ 30 \mathrm{ml}$ bone marrow (3). Numerous research groups have devoted their studies to the identification of a novel type of stem cell to replace BMSCs for bone repair. Skeletal muscle-derived stem cells (MDSCs) are a type of pluripotent stem cell with multiple differentiation potential and self-renewal ability. In addition, these stem cells are abundant, easy to acquire and rapidly amplified (4). The current study aimed to investigate the isolation and culture of MDSCs, as well as to verify their osteogenic potential in vitro. The results are likely to provide a basis for future clinical applications to promote bone healing in vivo.

\section{Materials and methods}

Isolation and culture of primary cells. The medial muscles from the hind limbs of New Zealand white rabbits were dissected ( 5.0 g) under sterile conditions (5). The study was approved by the ethics committee of the First Affiliated Hospital of Soochow University (Suzhou, China). Following careful removal of the surrounding connective tissue, adipose tissue and capillaries, the muscles were then sliced and ground into small sections using a scalpel blade. The minced muscles were then thoroughly washed three times with sterile phosphate-buffered saline (PBS) and digested with $2 \mathrm{~g} / 1$ type I collagenase (Invitrogen Life Technologies, Carlsbad, CA, USA) for $1 \mathrm{~h}$ at $37^{\circ} \mathrm{C}$ with $5 \% \mathrm{CO}_{2}$ and $85 \%$ humidity, as well as $2.5 \mathrm{~g} / 1$ trypsin (Invitrogen Life Technologies) for $40 \mathrm{~min}$. Following digestion, the cell suspension was centrifuged with an AllegraX-12R centrifuge (Beckman Coulter, Brea, CA, USA) at $200 \mathrm{x}$ g for $10 \mathrm{~min}$. The cells were then filtered with 70 - and $40-\mu \mathrm{m}$ cell strainers to successively break down any remaining cell clusters. Following further centrifugation (200 x $\mathrm{g}$ for $10 \mathrm{~min}$ ), the cell suspension was re-suspended in Dulbecco's modified Eagle's medium (DMEM; Invitrogen Life Technologies) containing 10\% fetal bovine serum (FBS), 10\% horse serum and $2 \%$ penicillin/streptomycin, prior to being plated onto a dish. Each dish was coded and cultured for $1 \mathrm{~h}$ in an incubator at $37^{\circ} \mathrm{C}$ with $5 \% \mathrm{CO}_{2}$ and $85 \%$ humidity. Since the cells that adhered rapidly within this one-hour incubation were predominantly fibroblasts, the supernatant was withdrawn and gently transferred to a new dish. This serial replating of the supernatant was repeated $\sim 5$ times. In order to prevent cell 
differentiation, cultures were maintained at semi-confluence and sub-cultured every 4-5 days with daily medium changes until adequate cell numbers were obtained.

Identification of MDSCs. Cells were seeded in 12-well culture plates and cultured in DMEM for $24 \mathrm{~h}$. When the cells had stretched, they were fixed with cold acetone for $5 \mathrm{~min}$ at $-20^{\circ} \mathrm{C}$. Following rinsing with PBS three times, the cells were incubated for $1 \mathrm{~h}$ at $37^{\circ} \mathrm{C}$. Anti-desmin (Sigma, St Louis, MO, USA) and anti-myosin antibodies (Invitrogen Life Technologies) were used as primary antibodies and were diluted in PBS. A secondary antibody was applied for $1 \mathrm{~h}$ at room temperature. Cells were rinsed with PBS and incubated at room temperature with Hoechst 33342 fluorescent solution (Thermo Fisher Scientific Pierce, Rockford, IL, USA) for $5 \mathrm{~min}$, followed by visualization using fluoroscopy (AXIOVERT 40C; Carl Zeiss AG, Oberkochen, Germany). Positive cells were visualized in green, while the nucleus was visualized in blue.

Cells $\left(5 \times 10^{5}\right)$ were re-suspended and incubated at $4^{\circ} \mathrm{C}$ for 30 min with $100 \mu 1$ PBS containing $2 \mu 1$ anti-CD105-phycoerythrin antibody (Sigma-Aldrich). Following the washing process, unconjugated primary antibodies were incubated with a secondary goat anti-mouse IgG-FITC (Santa Cruz Biotechnology, Inc., Dallas, TX, USA) for an additional $15 \mathrm{~min}$ at $4^{\circ} \mathrm{C}$. The cell suspension was then washed with PBS and labeled cells were analyzed by flow cytometry (AllegraX-12R; Beckman Coulter). Control samples were incubated with PBS instead of primary antibody, followed by incubation with secondary antibody.

Osteogenic differentiation. MDSCs were seeded in 6-well plates at a density of $10^{6}$ cells/well and cultured in osteogenic medium containing DMEM with $20 \%$ FBS, $0.1 \mathrm{mM}$ dexamethasone, $0.05 \mathrm{mM}$ ascorbic acid-2-phosphate, $10 \mathrm{mM}$ $\beta$-glycerolphosphate, $100 \mathrm{U} / \mathrm{ml}$ penicillin and $100 \mathrm{U} / \mathrm{ml}$ streptomycin. The cells cultured in basic medium served as control and culture medium was changed every three days.

Assessment of cell proliferation. Cell proliferation was quantitatively evaluated by 3-(4,5-dimethylthiazol-2-yl)-2,5-diphenyl tetrazolium bromide (MTT) assay. MDSCs were seeded in 96-well plates in medium containing 10\% FBS at a density of 5,000 cells/well. The MTT assay was performed in triplicate. MTT $(20 \mu \mathrm{l})$ was added to a final concentration of $5 \mathrm{mg} / \mathrm{ml}$ and the reaction mixture was incubated for $4 \mathrm{~h}$ at $37^{\circ} \mathrm{C}$. The supernatant was removed and $150 \mu \mathrm{l}$ DMSO was added to each well to solubilize the water-insoluble purple formazan crystals. The absorbency was measured at a wavelength of $590 \mathrm{~nm}$.

Alkaline phosphatase (ALP) assay. MDSCs were seeded in 12 -well plates and covered with medium. Following $48 \mathrm{~h}$, the medium was removed and ALP staining was performed. All steps were performed in accordance with the manufacturer's instructions (Jiancheng Biotech, Guangzhou, China). The staining was observed via microscope following four, eight, 12 and 16 days of differentiation. The ALP-positive cells stained brown. For each experiment, a minimum of three plates were counted and the experiments were repeated three times.

MDSCs were also seeded in 48-well plates and incubated in medium. ALP activity was measured following four, eight, 12 and 16 days of differentiation by the ALP assay kit (BioVision, Inc., San Francisco, CA, USA). The cells were harvested with lysis buffer. Total protein concentrations of supernatant were determined by the Bradford method. An aliquot $(10 \mu \mathrm{l})$ of supernatant was added to $100 \mu 150 \mathrm{mM}$ p-nitrophenylphosphatase hexahydrate containing $1 \mathrm{mM}$ $\mathrm{MgCl}_{2}$ and the mixture was incubated at $37^{\circ} \mathrm{C}$ for $30 \mathrm{~min}$. Absorption at $405 \mathrm{~nm}$ was measured with a spectrophotometer. ALP activity per total protein $(\mu \mathrm{g})$ represented the millimoles of p-nitrophenol released following $30 \mathrm{~min}$ incubation at $37^{\circ} \mathrm{C}$.

Assay of mineralization. The mineralization of MDSCs was determined in 12-well plates using Alizarin red staining (ScienCell Research Laboratories, Carlsbad, CA, USA) following four, eight, 12 and 16 days of differentiation. Following two PBS washes, the cells were fixed with ice-cold 70\% ethanol for 10 min and stained with 0.1\% Alizarin Red in Tris- $\mathrm{HCl}$ buffer solution (adjusted to $\mathrm{pH} 8.3$ ) for $30 \mathrm{~min}$ at $37^{\circ} \mathrm{C}$. Following staining, MDSCs were rinsed three times with distilled water followed by $70 \%$ ethanol.

Evaluation of osteogenic potential by quantitative polymerase chain reaction ( $q P C R$ ). Osteogenic gene expression (osteocalcin and osteopontin) was quantified by qPCR. Total RNA was extracted with TRIzol reagent (Invitrogen Life Technologies) from MDSCs, according to the manufacturer's instructions. cDNA synthesis was performed with $2 \mu \mathrm{g}$ total RNA. qPCR was performed using the ABI Prism 7500 sequence-detection system (Applied Biosystems, Foster City, CA, USA) with SYBR-Green, according to the manufacturer's instructions. For each sample of DNA, two test runs were performed; one used the osteogenic gene primers and the other used the GAPDH gene primers (Table I). Each running sample was composed of $3 \mu \mathrm{l} \mathrm{cDNA}, 1 \mu \mathrm{l}$ forward primer, $0.25 \mu \mathrm{l}$ reverse primer and $5 \mu \mathrm{l}$ SYBR-green. Each cycle (40 cycles in total) included denaturation at $50^{\circ} \mathrm{C}$, annealing at $95^{\circ} \mathrm{C}$ and elongation at $60^{\circ} \mathrm{C}$. Relative gene expression was normalized against GAPDH expression and the data were presented as the fold change using the formula $2^{-\Delta \Delta C T}$, as recommended by the manufacturer.

\section{Results}

Characterization of MDSCs. The number of adhered MDSCs was reduced within two to three days. The cells assumed a spindle-shaped fibroblastic appearance, proliferated rapidly and became confluent at one week of culture. Immunohistochemical staining demonstrated that the majority of detected cells expressed myogenic markers (desmin and myosin; Fig. 1). Expression of desmin is ubiquitous throughout the whole cell except for the nucleus, while the expression of myosin is localized in the nucleus. Flow cytometric analysis also demonstrated that the majority of cells were positive for the MSC marker, CD105 (Fig. 2). When plated and cultured in osteogenic medium, MDSCs changed from an elongated fibroblastic appearance to a more rounded, cuboidal shape and formed an extensive network of dense, multilayered nodules.

Assessment of cell proliferation. Based on the MTT assay, growth curves of differentiated and undifferentiated MDSCs 
Table I. Primers used in quantitative polymerase chain reaction.

\section{Gene}

Osteocalcin

Osteopontin

GAPDH
Primer sequence

Forward: 5'-CCGGGAGGAGATCTG;TGAAA-3' Reverse:5'-CTGCCTTCTTCCACAATTTTATCC-3'

Forward: 5'-GCCAGTTGCAGCCTTCTCA-3' Reverse: 5'-GCCATGCCCAAGAGACAAAA-3' Forward: 5'-CGGACACGGACAGGATTGAC-3' Reverse: 5'-CCAGACAAATCGCTCCACCA-3'
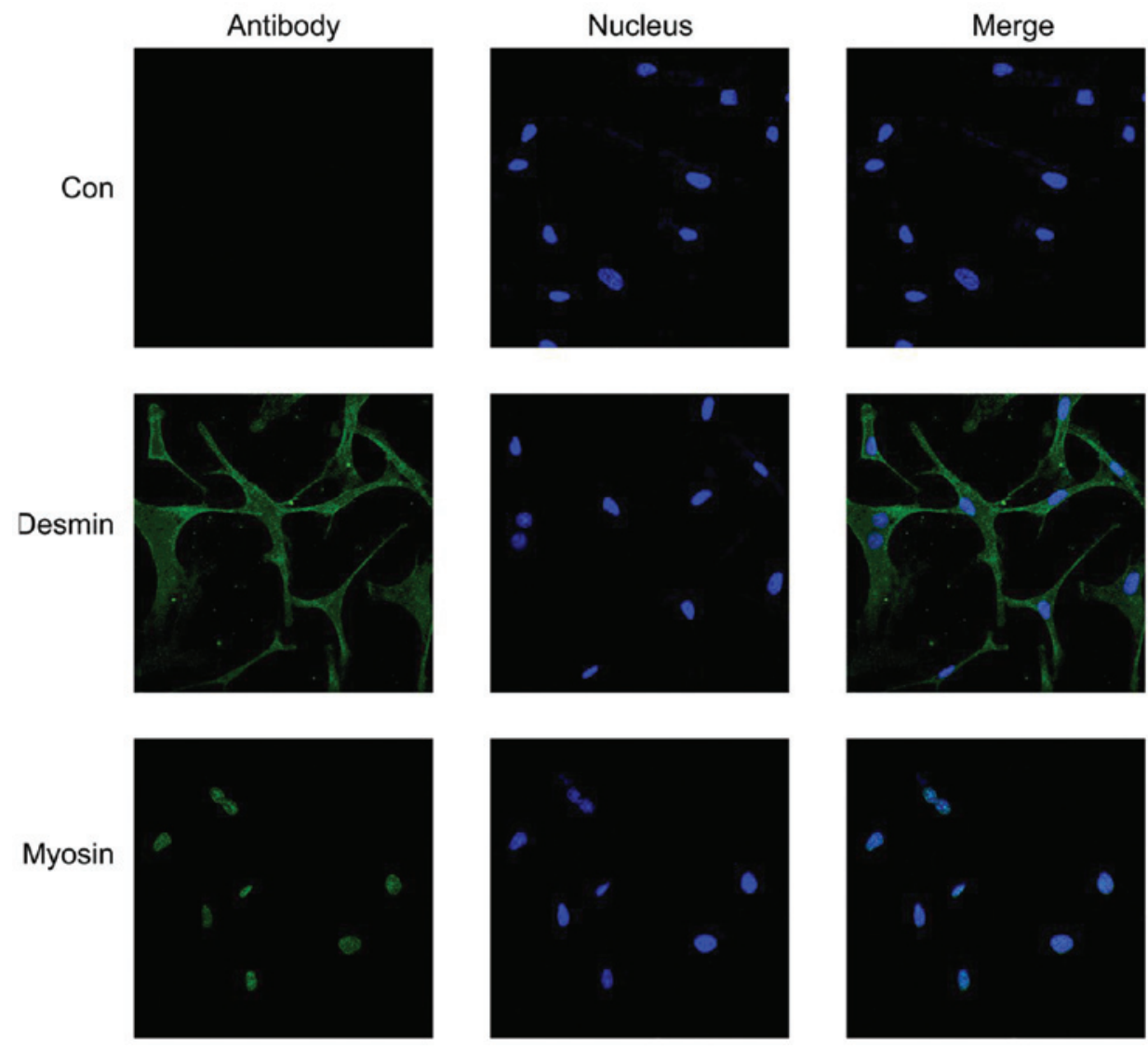

Figure 1. Immunohistochemical staining of desmin and myosin in muscle-derived stem cells (magnification, $\mathrm{x} 400$ ). Green fluorescence displayed the signals of corresponding protein expression. Blue fluorescence displayed a nucleus counterstained by Hoechst 33342. Dilution ratios: Desmin, 1:100; myosin, 1:25.

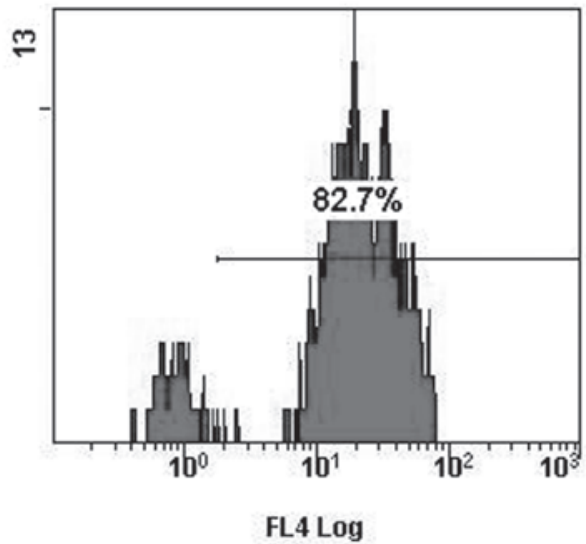

Figure 2. Flow cytometric analysis of CD105 in muscle-derived stem cells. The majority of cells were positive for CD105. presented an ' $\mathrm{S}$ ' shape. The lag phase occurs at $24 \mathrm{~h}$, followed by the logarithmic phase of growth and a growth-arrested phase. No significant difference was observed on day four following osteogenic differentiation. However, between days eight and 16, the cell proliferation rate of differentiated MDSCs was significantly higher than that of undifferentiated MDSCs $(\mathrm{P}<0.05)$. This growth curve shows significantly higher cell growth rates in the differentiated MDSC group than in the undifferentiated MDSC group ( $\mathrm{P}<0.05$; Fig. 3 ), indicating that the differentiated MDSC population proliferated faster than the undifferentiated MDSCs population.

ALP assay. The differentiated MDSCs stained positive for membrane-bound ALP on day four and the staining became stronger at day 16 (Fig. 4). Results indicated that osteogenic 


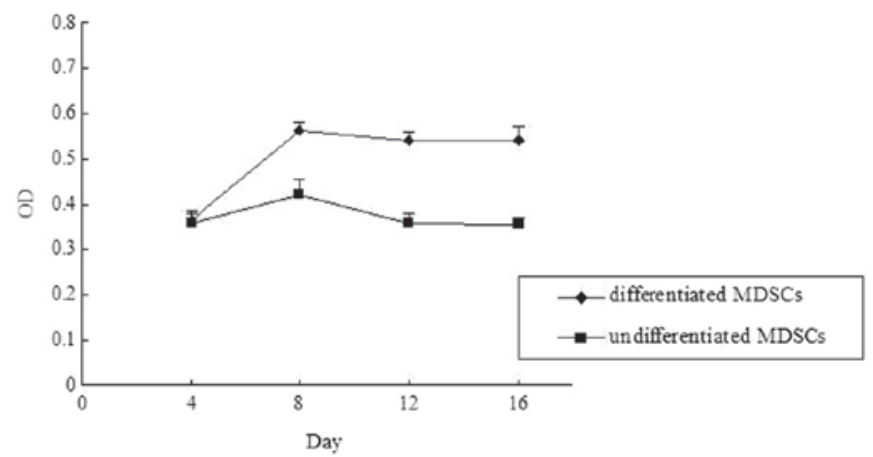

Figure 3. MTT assay for cell proliferation. The differentiated MDSC population underwent proliferation faster than the undifferentiated MDSC population. MDSC, muscle-derived stem cell; MTT, 3-(4,5-Dimethylthiazol-2-yl)-2,5-diphenyl tetrazolium bromide.

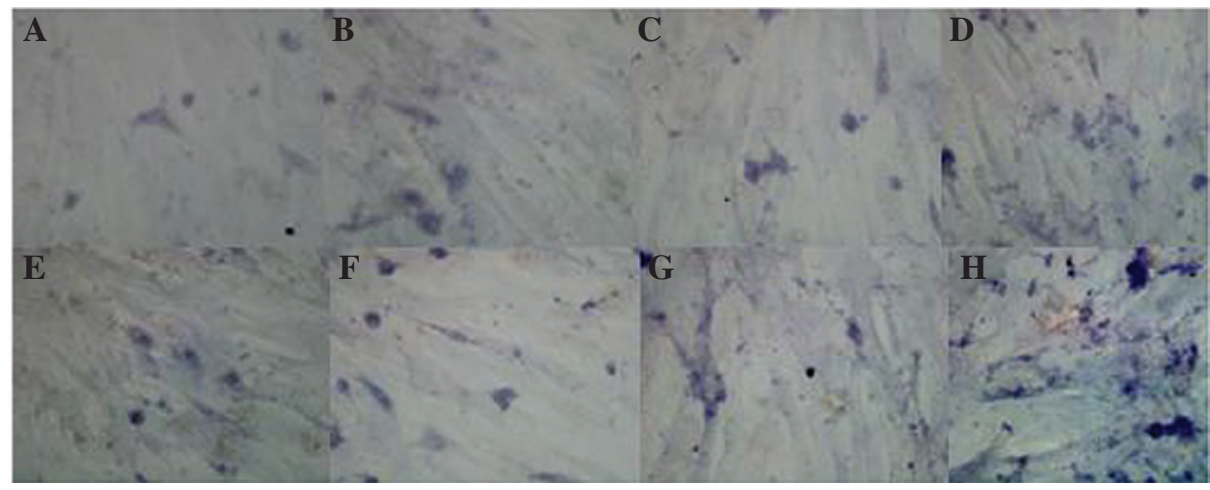

Figure 4. Alkaline phosphatase staining (magnification, x100). Undifferentiated MDSCs at (A) four, (B) eight, (C) 12 and (D) 16 days. Differentiated MDSCs at (E) four, (F) eight, (G) 12 and (H) 16 days. MDSC, muscle-derived stem cell.

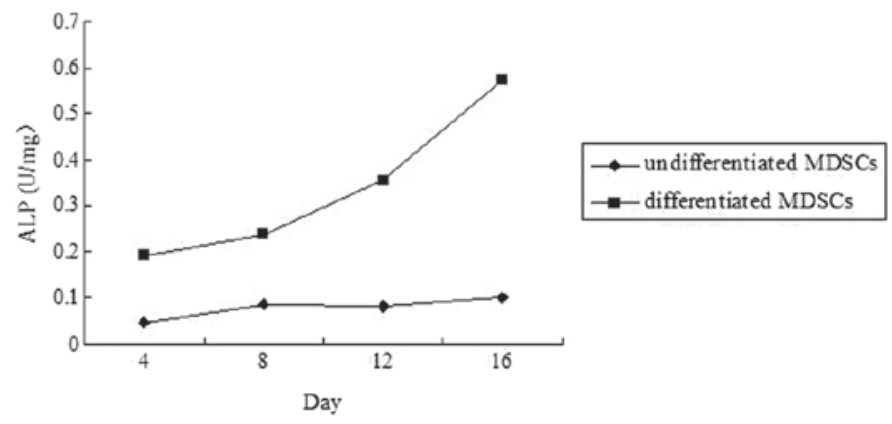

Figure 5. Assay of ALP activity. Quantitation of ALP activity demonstrated that the ALP activity of undifferentiated MDSCs was lower than that of corresponding differentiated MDSCs. ALP, alkaline phosphatase; MDSC, muscle-derived stem cell.

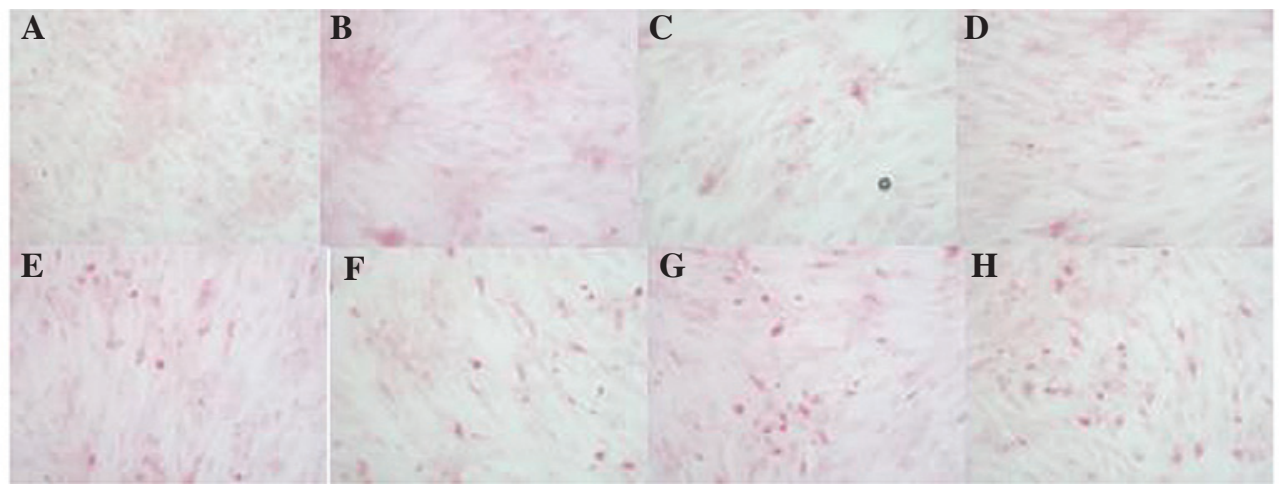

Figure 6. Alizarin red staining (magnification, x100). Undifferentiated MDSCs at (A) four, (B) eight, (C) 12 and (D) 16 days. Differentiated MDSCs at (E) four, (F) eight, (G) 12 and (H) 16 days. MDSC, muscle-derived stem cell. 
A
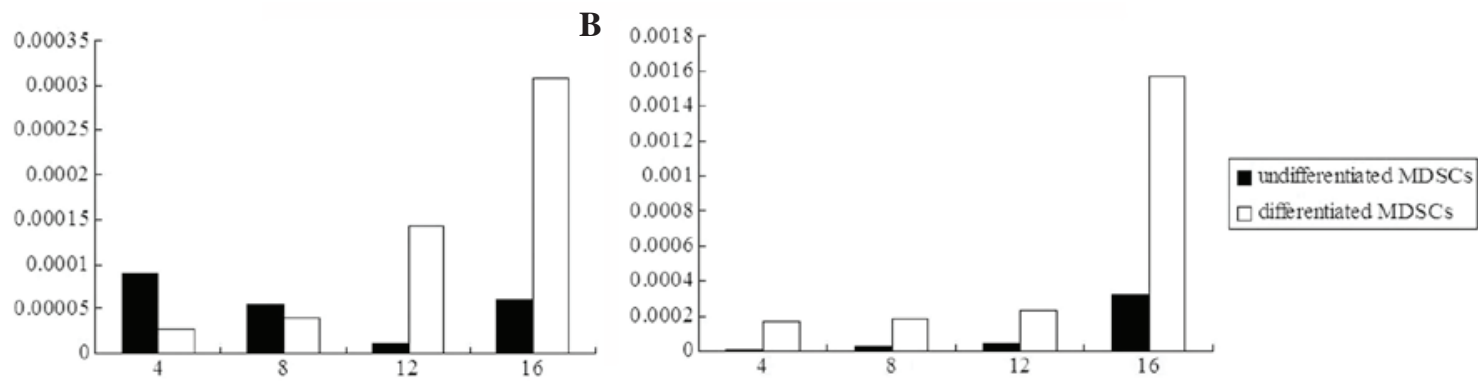

Figure 7. qPCR evaluation of mRNA expression during MDSC differentiation. Relative mRNA levels were normalized against GAPDH levels. Osteogenic genes in the differentiated cells increased gradually and attained significantly higher levels of (A) osteocalcin and (B) osteopontin. MDSC, muscle-derived stem cell; qPCR, quantitative polymerase chain reaction.

differentiation stimulated the MDSCs to undergo osteogenic differentiation, as judged by the production of ALP (an early osteogenic marker) in response to osteogenic differentiation. By contrast, undifferentiated MDSCs predominantly showed negative reactions to ALP staining at any time point, demonstrating that undifferentiated MDSCs did not undergo osteogenic differentiation. Quantitation of ALP activity demonstrated that the ALP activity of undifferentiated MDSCs was significantly lower than that of corresponding differentiated MDSCs. ALP activities of differentiated MDSCs increased between days four and 12. As time progressed, the activity was higher, reaching a peak at day 16 . By comparison, the activities of undifferentiated MDSCs remained stable at a low level (Fig. 5).

Mineralization assay. The well-defined mineralization nodules stained by alizarin red staining were observed in differentiated MDSCs. Between days four and 12, Alizarin red $(+)$ nodules increased constantly. At $\sim$ day 16, the progress of matrix mineralization significantly step by step diffusely spread. However, no marked mineralization nodules were found in undifferentiated MDSCs at any time point (Fig. 6).

Analysis of the mRNA expression of osteocalcin and osteopontin. Osteogenic differentiation had a clear positive effect on the expression of two bone-associated proteins, osteocalcin and osteopontin, in MDSCs and this expression was markedly increased over time. The synthesis and secretion of osteocalcin and osteopontin from differentiated MDSCs increased gradually and attained a significantly higher level at day 16, while the secretion of undifferentiated MDSCs was quite low and stable at each time point (Fig. 7).

\section{Discussion}

Skeletal muscle has been extensively investigated as a potential source for isolation of several stem cells over the past few years (6). Satellite cells located beneath the basal lamina of mature skeletal muscle fibers, often referred to as 'muscle stem cells', have long been considered monopotential stem cells capable of only giving rise to cells of the myogenic lineage (7). MDSCs, which may represent a predecessor of the satellite cell, are considered to be distinct in that they may not be restricted to the myogenic lineage or mesenchymal tissues and are able to differentiate into multiple lineages (8).
Several studies have hypothesized that there are at least two types of stem cells in skeletal muscle tissue; the typical spindle muscle satellite cells and round MDSCs (9). Muscle satellite cells are myogenic precursors, which are capable of regenerating skeletal muscle and demonstrate self-renewal properties; however, they are considered to be committed to the myogenic lineage (10). MDSCs are precursors of muscle satellite cells and have a stronger capacity to proliferate and broader potential of differentiation compared with muscle satellite cells. These cells may differentiate into more specialized cells with morphological and phenotypic features of cells including neurocytes, hepatocytes, endotheliocytes, neurogliocyte, skeletal muscle cells. MDSCs not only differentiate into mesodermal cells, but also endodermal cells. By breaking the limit of the germ layers, these cells exhibit wide application prospects $(11,12)$.

Primary culture methods of MDSCs include the tissue block method and digestion methods, of which the enzyme digestion culture method is the most commonly used (8-10). In the present study, mechanical shearing and enzyme digestion was used to break the MDSC membrane and remove the connective tissues interfering with cell growth. MDSCs dispersed into monocellular suspension contribute to nutrient absorption and excretion of metabolites from the medium. Since the mechanism of action and the optimal condition of various types of digestive enzymes are not all the same, stepwise digestion was performed using collagenase combined with trypsin, which yielded 80-90\% MDSCs. In addition, MDSCs were purified by removing muscle fiber fragments and nonmyogenic components in cell suspension liquid, in order to meet the requirements for the next step. A suitable centrifugation method ( $200 \mathrm{x} \mathrm{g}$; $3 \mathrm{~min}$ ) was used to precipitate the muscle fiber fragments. If the centrifugal force is too large or the centrifugation time is too long, MDSCs are likely to be damaged and cell yield decreased. However, if the centrifugation time is too short, nonmyogenic components mix with cells. In addition, cell strainers of various sizes were also used to filter muscle fiber fragments. For removal of the nonmyogenic components in mixed cell suspension, the preplate technique was mainly utilized, which isolates cell populations based on their differential adhesion characteristics. The adherent ability of nonmyogenic components is stronger than that of the MDSCs and the majority of early adherent cells are nonmyogenic cells. Non-adherent cell suspensions were removed and seeded in novel culture dishes and this preplate 
procedure was repeated until MDSCs were pure. Regardless of certain disadvantages, for example a time consuming (five to seven days for adherence) and complex procedure, as well as a high possibility of pollution, cell damage is minimal and cell purity may reach $>80 \%$ using this technique (13).

There is no generally established specific marker for identification of multipotential stem cells from skeletal muscle. Markers commonly used are expressed in various tissues and with the prolongation of cell culture time, the cell behaviors and surface markers are likely to have changed; thus rendering the identification of MDSCs difficult. In the present study, a combination of specific generally accepted molecular markers, including stem cells and myogenic cells, was optimized to identify MDSCs. CD105, a characteristic glycoprotein expressed on stem cells, has frequently been used as a relatively specific marker for the identification of mesenchymal stem cells. Desmin is a major essential intermediate filament protein, as well as a specific marker for muscle tissue and is used to distinguish between myogenic and nonmyogenic tissues (14). The positive rate of desmin may reach $90 \%$ in MDSCs, while in fibroblast and smooth muscle cells, the rate of desmin-positive cells is only $15 \%$ (15). Myosin is the most common protein in muscle cells and is a globulin responsible for the elastic and contractile properties of muscle, combining with actin to form actomyosin. It forms the thick filament of myofibrilla and is the most abundant protein expressed in skeletal muscle, making up $25 \%$ of the total protein database (16). Based on this combination, preliminary identification may be performed successfully.

MDSCs differentiated into multinucleated myotubes and muscle fibers autonomously without any induction; in the myoblast differentiation medium, they differentiated into myogenic cells, including skeletal muscle cells, smooth muscle cells and cardiocytes (17-20). Jackson et al (21) injected MDSCs to six lethally irradiated mice. Following 12 weeks, all mice showed high-level engraftment of muscle-derived cells representing all predominant adult blood lineages. This indicated that cells derived from skeletal muscle generate the major hematopoietic lineages. In the present study, following culture with osteogenic differentiation medium, MDSCs were positive for ALP and mineralized nodule staining. Immunohistochemical staining and flow cytometric analysis showed that desmin, myosin and CD105 were also positive in the differentiated MDSCs, indicating that MDSCs had differentiated into osteogenic cell lines. Although the mechanism of differentiation is not yet clear, it may be that MDSCs directly differentiate into osteogenic precursor cells or there were other stem cells in skeletal muscle that differentiated into other osteoblasts. When these precursor cells are mature, they may express various specific markers of bone tissues. Following osteogenic differentiation, the MDSCs in the present study gradually changed in morphology and eventually formed round cells with a large body. In addition, expression of ALP, osteocalcin and osteopontin was noted in these cells. ALP is one of the early marker enzymes of maturity of extracellular matrix and hydrolyzes organic phosphatase, increases the local concentration of phosphoric acid, destroys inhibitors of calcification and initiates the calcification process for promoting mineralization of the extracellular matrix. ALP expression was detected four days following osteogenic differentiation in the present study and expression was significantly increased following day 16. Osteopontin is a key factor of maturity of the extracellular matrix and is associated with hydroxyapatite deposition of extracellular matrix; it is important in mineralization metabolism and reconstruction of bone. Osteocalcin is a type of noncollagenous bone matrix protein secreted by mature osteoblasts. It regulates the speed and direction of calcium deposition, as a key factor of bone formation. In the present study, the mRNA expression of these two proteins was less in undifferentiated MDSCs; however, when the cells were cultured in the osteogenic differentiation medium, expression was higher than previously observed. This indicated that MDSCs had differentiated into osteoblasts.

In the present study, MDSCs were successfully isolated and cultured from rabbit skeletal muscle tissues, and in vitro analyses indicated that the cells were capable of differentiating into osteoblasts. Since the concept of cell therapy has already been introduced into the clinical setting, MDSCs which represent a valuable source of osteoprogenitors, exhibit the potential for the repair of skeletal damage due to injury or disease. Further studies are required in order to identify optimization of cell isolation and expansion, as well as the control of cell differentiation and transformation in various environments.

\section{Acknowledgements}

This study was supported by grants from the National Natural Science Foundation of China (grant no. 81101370), Jiangsu Science and Technology Support Program (Social Development) (grant no. BE2011672) and Jiangsu Collegiate Natural Science Foundation (grant no. 12KJB320008).

\section{References}

1. Strioga M, Viswanathan S, Darinskas A, Slaby O and Michalek J: Same or not the same? Comparison of adipose tissue-derived versus bone marrow-derived mesenchymal stem and stromal cells. Stem Cells Dev 21: 2724-2752, 2012.

2. Osugi M, Katagiri W, Yoshimi R, Inukai T, Hibi H and Ueda M: Conditioned media from mesenchymal stem cells enhanced bone regeneration in rat calvarial bone defects. Tissue Eng Part A 18: 1479-1489, 2012.

3. Mauney JR, Kirker-Head C, Abrahamson L, Gronowicz G, Volloch V and Kaplan DL: Matrix-mediated retention of in vitro osteogenic differentiation potential and in vivo bone-forming capacity by human adult bone marrow-derived mesenchymal stem cells during ex vivo expansion. J Biomed Mater Res A 79: 464-475, 2006.

4. Schindeler A, Liu R and Little DG: The contribution of different cell lineages to bone repair: exploring a role for muscle stem cells. Differentiation 77: 12-18, 2009.

5. Usas A, Ho AM, Cooper GM, Olshanski A, Peng H and Huard J: Bone regeneration mediated by BMP4-expressing muscle-derived stem cells is affected by delivery system. Tissue Eng Part A 15: 285-293, 2009.

6. Shi H, Verma M, Zhang L, Dong C, Flavell RA and Bennett AM: Improved regenerative myogenesis and muscular dystrophy in mice lacking Mkp5. J Clin Invest 123: 2064-2077, 2013.

7. Yan J, Gan L, Yang H and Sun C: The proliferation and differentiation characteristics of co-cultured porcine preadipocytes and muscle satellite cells in vitro. Mol Biol Rep 40: 3197-3202, 2013.

8. BuenoDF, Kerkis I,Costa AM, et al: New source of muscle-derived stem cells with potential for alveolar bone reconstruction in cleft lip and/or palate patients. Tissue Eng Part A 15: 427-435, 2009.

9. Asakura A: Stem cells in adult skeletal muscle. Trends Cardiovasc Med 13: 123-128, 2003.

10. Jankowski RJ, Deasy BM and Huard J: Muscle-derived stem cells. Gene Ther 1: 642-647, 2002. 
11. Wu X, Wang S, Chen B and An X: Muscle-derived stem cells: isolation, characterization, differentiation, and application in cell and gene therapy. Cell Tissue Res 340: 549-567, 2010.

12. Arsic N, Mamaeva D, Lamb NJ and Fernandez A Muscle-derived stem cells isolated as non-adherent population give rise to cardiac, skeletal muscle and neural lineages. Exp Cell Res 314: 1266-1280, 2008.

13. Huard J, Yokoyama T, Pruchnic R, Qu Z, Li Y, Lee JY Somogyi GT, de Groat WC and Chancellor MB: Muscle-derived cell-mediated ex vivo gene therapy for urological dysfunction. Gene Ther 9: 1617-1626, 2002.

14. Cornelison DD and Wold BJ: Single-cell analysis of regulatory gene expression in quiescent and activated mouse skeletal muscle satellite cells. Dev Biol 191: 270-283, 1997.

15. Wei Y, Li Y, Chen C, Stoelzel K, Kaufmann AM and Albers AE Human skeletal muscle-derived stem cells retain stem cell properties after expansion in myosphere culture. Exp Cell Res 317: 1016-1027, 2011.

16. Schiaffino $\mathrm{S}$ and Reggiani C: Myosin isoforms in mammalian skeletal muscle. J Appl Physiol 77: 493-501, 1994.
17. Lee JY, Cannon TW, Pruchnic R, Fraser MO, Huard J and Chancellor MB: The effects of periurethral muscle-derived stem cell injection on leak point pressure in a rat model of stress urinary incontinence. Int Urogynecol J Pelvic Floor Dysfunct 14: 31-37, 2003.

18. Reinecke H, Poppa V and Murry CE: Skeletal muscle stem cells do not transdifferentiate into cardiomyocytes after cardiac grafting. J Mol Cell Cardiol 34: 241-249, 2002.

19. Herreros J, Prósper F, Perez A, Gavira JJ, Garcia-Velloso MJ, Barba J, et al: Autologous intramyocardial injection of cultured skeletal muscle-derived stem cells in patients with non-acute myocardial infarction. Eur Heart J 24: 2012-2020, 2003.

20. Iijima Y, Nagai T, Mizukami M, Matsuura K, Ogura T, Wada $\mathrm{H}$, Toko $\mathrm{H}$, Akazawa $\mathrm{H}$, Takano $\mathrm{H}$, Nakaya $\mathrm{H}$ and Komuro I: Beating is necessary for transdifferentiation of skeletal muscle-derived cells into cardiomyocytes. FASEB J 17: 1361-1363, 2003

21. Jackson KA, Mi T and Goodell MA: Hematopoietic potential of stem cells isolated from murine skeletal muscle. Proc Natl Acad Sci USA 96: 14482-14486, 1999. 